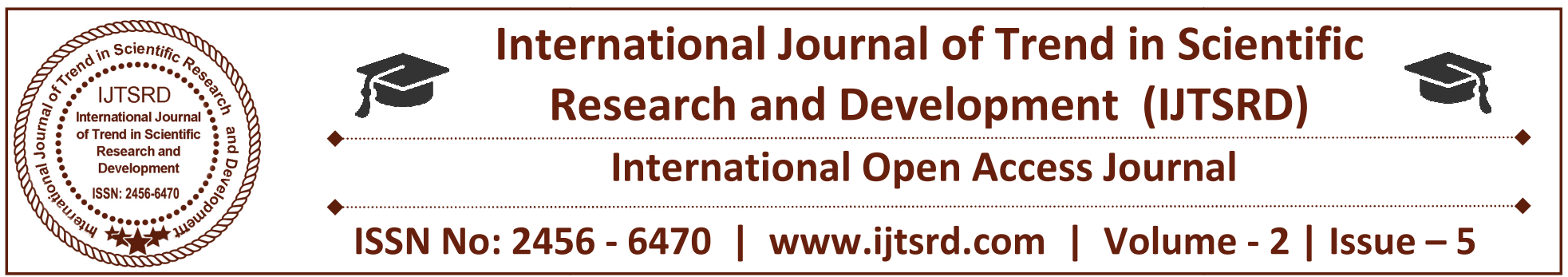

\title{
Survey of Detecting Heartbeats, Temperature and ECG of Human Body using IOT
}

\author{
Krishna B. Jariwala*, Prof. Jaimeel Shah \\ "Student \\ Department of Computer Science \& Engineering, \\ Parul Institute of Engineering and Technology, Vadodara, Gujarat, India
}

\begin{abstract}
In this paper, to presents an overview of the "SURVEY OF DETECTING HEARTBEATS, TEMPERATURE and ECG OF HUMAN BODY USING IOT". Smart health care means to monitor people's health outside the clinical settings. With the development of technology, we can digitally sensing body temperature, pulse rate and ECG using NodeMcu. Mainly NodeMcu is used because it has in built Wi-Fi module. The microcontroller on the board is programmed using the Arduino programming language". DS18B20 is used for the sense body temperature. Body temperature is a basic parameter for monitoring and diagnosing human health. Pulse sensor is used for sensing heart rate. ECG sensor is use to sense ECG of body. This device will allow measuring their mean arterial pressure (MAP) in about one minute and the all parameters will be displayed on the Android application. The system can be used to measure physiological parameters, such as Heart rate and Body Temperature.
\end{abstract}

Keyword: NodeMcu, Body Temperature, Heart rate, ECG of body, Sensors.

\section{INTRODUCTION}

In this paper, we solve the problem regarding human health. We can detect body temperature, heart rate and ECG of human body using smart healthcare system. In the new era of communication and technology, the explosive growth of electronic devices, smart phones and tablets which can be communicated physically or wirelessly has become the fundamental tool of daily life. The next generation of connected world is Internet of Things (IOT) which connects devices, sensors, appliances, vehicles and other "things". The things or objects may include the radio-frequency identification (RFID) tag, mobile phones, sensors, actuators and much more. With the help of IOT, we connect anything, access from anywhere and anytime, efficiently access any service and information about any object. The aim of IOT is to extend the benefits of Internet with remote control ability, data sharing, constant connectivity and so on. Using an embedded sensor which is always on and collecting data, all the devices would be tied to local and global networks.

The IOT technology can provide a large amount of data about human, objects, time and space, while combining the current Internet technology and IOT provides a large amount of space and innovative service based on low-cost sensors and wireless communication. IPv6 and Cloud computing promote the development of integration of Internet and IOT. It is providing more possibilities of data collecting, data processing, port management and other new services. Every object which connects to IOT requires a unique address or identification with IPv6.

There are so many people in the world whose health may suffer because they do not have proper access to hospitals and health monitoring. Due to the latest technology, small wireless solutions which are connected to IOT can make it possible to monitor patients remotely instead of visiting the physical hospital. A variety of sensors likes; DS18B20, Heart rate sensor, ECG sensor which are attached to the body of a patient can be used to get health data securely, and the collected data can be analyzed and sent to the server using different transmission media [1]. 


\section{Related Work}

Nowadays there are many existing smart patient monitoring systems which are quite famous and easily available. Android app integrated with Android wearable device which monitors user's health and responds accordingly.

Security issue in Wearable device is one of the major reason due to which we are proposing a more secure system. Many zero-day vulnerabilities are present in Wearable devices which increases the risk in using such system. Tampering with patient's health related data may cause serious and sometimes even life threatening issues. Overheating is another problem with Wearable device which may reflect changes in temperature sensor's values and can give wrong data. Also there is unreliability problem in many Wearable devices.

There is lack of compatibility between hardware support and Android stock OS or modified OS. Nowadays, many fitness monitoring devices are available in markets which are capable of monitoring user's fitness level and notifying him / her accordingly. But measuring only physical fitness is not sufficient, continuous health monitoring is also needed for better living. There are many embedded systems[2] present which monitor either temperature or pulse rate or ECG of user for better notification, but measuring combination of all will get us a more detail and precise information about person's current health condition.

Existing systems provide only notification about fitness and health conditions but report of daily and monthly variations is needed for better diagnosis which is absent in many of the existing systems. They are not providing user current location [3].

\section{Proposed Work}

Health monitoring is important to be checked regularly to make sure our body constantly maintain in healthiness and excellent condition. This system observed for health monitoring such as Heart Rate (HR), temperature and ECG. These parameters will interpret some important information regarding human health. For example; high temperatures indicate someone having fever while unstable heart rate is sign to heart problem. ${ }^{[1]}$ After mapping parameters, it first compare them to our pre-dataset. If collected data are abnormal i.e. current data are not matching with our data set, it sends notification to the doctor or relative and inform them that "particular user is not well" and also send the user's current location. These all data will store in user's profile and make monthly report of particular user's health. All result will display on android application. Such way we can easily monitoring on our and family members health.

\section{A. Proposed Algorithm}

Step 1: Initializing NodeMcu, let us consider; DS18B20 as bt, Heart rate sensor as an hr, ECG sensor as es

Step 2: Connect bt with NodeMcu and other part of bt connect with human body.

Step 3: To calculate temperature of human body; First Temperature is calculated in Arduino and that value display on android application. After that all values store in to database of system. bt is calculate Body temperature in every 1hour. If temperature will abnormal (i.e. $>=98.6 \mathrm{f}<=$ ) then alert message send to the relatives of doctor.

\section{temp $=$ analog*0.322265625; \\ tempf $=($ temp $* 1.8)+32$}

Step 4: Now, Connect hr with NodeMcu and another side of sensor is connected with human body.

Step 5: Before connection of hr, connect multiplexer with NodeMcu. We know that NodeMcu gives only one analog output at a one time. We use multiple sensors in this system. Now connect hr with NodeMcu and another side of hr will connect with human body.

Step 6: After connection, calculate the Heart rate of Human body. If pulse will abnormal then send the alert message through android application to relatives or doctor with user current location. And store all values after 1 hour in database.

Step 7: Heart rate calculated and display depend on human age;

If age between 10-20 year; $\{\mathrm{hr}>100$ $170 \mathrm{bpm}\}$

If age between 30-40 year; $\{\mathrm{hr}>180$ 190bpm

If age between 41-55 year; $\{\mathrm{hr}>175-$ $165 \mathrm{bpm}\}$

If age between 60-70 year; $\{\mathrm{hr}>160$ $150 \mathrm{bpm}\}$ 
Step 8: Now connect ES with NodeMcu (esp8266) and same as other sensor another end of the device will connect with human body.

Step 9: Repeat step 6 for ES.

Step 10: Give history of User.

Step 11: Stop.

\section{B. System Flow}

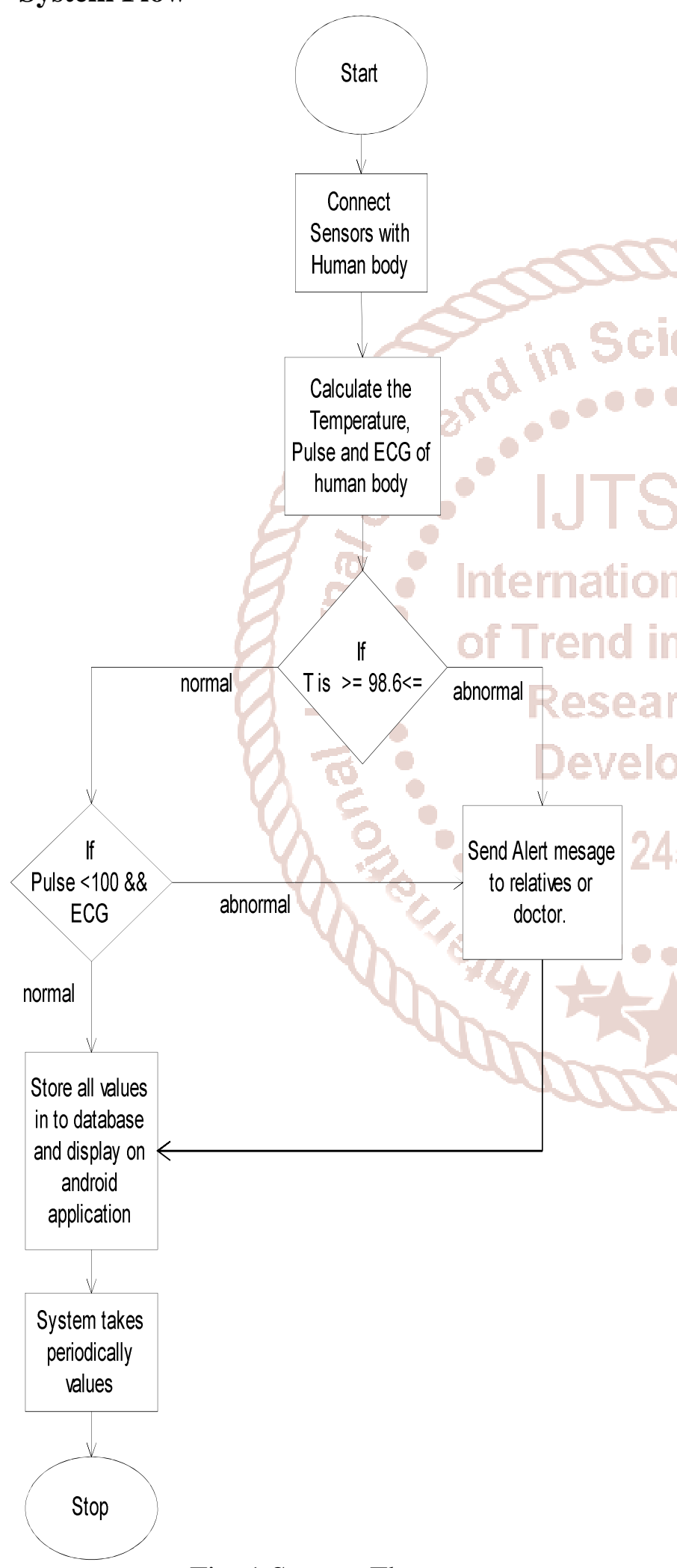

Fig. 1 System Flow
IV. HARDWARE

A. Node Mcu (ESP8266)

1. Features:

$>$ Integrated TCP/IP protocol stack

$>$ Supports antenna diversity

$>$ Wi-Fi $2.4 \mathrm{GHz}$, support WPA/WPA2

$>$ Wake up and transmit packets in $<2 \mathrm{~ms}$

$>$ Standby power consumption of $<1.0 \mathrm{~mW}$ (DTIM3)

$>+20 \mathrm{dBm}$ output power in $802.11 \mathrm{~b}$ mode

$>$ Operating temperature range: $40 \mathrm{C} \sim 125 \mathrm{C}$ [7].

2. Parameters:

$>$ Wi-Fi Protocles: 802.11 b/g/n

Frequency Range: $2.4 \mathrm{G}-2.5 \mathrm{G} \quad$ (2400M2483.5M)

Operating Voltage: $3.0 \sim 3.6 \mathrm{~V}$

Temperature Package Size: $5 \times 5 \mathrm{~mm}$

External Interface: N/A

$>$ Wi-Fi mode: station/softAP/SoftAP+station Security: WPA/WPA2

Network Protocols:

IPv4, TCP/UDP/HTTP/FTP [7].

3. Applications

Home Appliances

Sensor Networks

Wearable Electronics

Wi-Fi Location-aware Devices

Wi-Fi Position System Beacons [7].

B. $=$ LM35

1. Features

$>$ Linear $+10-\mathrm{mV} /{ }^{\circ} \mathrm{C}$ Scale Factor

$>$ Low-Cost Due to Wafer-Level Trimming

$>$ Operates From $4 \mathrm{~V}$ to $30 \mathrm{~V}$

$>$ Less Than 60- $\mu \mathrm{A}$ Current Drain

$>$ Low Self-Heating, $0.08^{\circ} \mathrm{C}$ in Still Air

$>$ Non-Linearity Only $\pm 1 / 4{ }^{\circ} \mathrm{C}$ Typical

$>$ Low-Impedance Output, $0.1 \Omega$ for 1-mA Load [8].

\section{Pulse Sensor}

Pulse Sensor is a well-designed plug-and-play heartrate sensor. It can be used by students, artists, athletes, makers, and game \& mobile developers who want to easily incorporate live heart rate data into their projects. The sensor clips onto a fingertip or earlobe and plugs right into Arduino or NodeMcu with some jumper cables. It also includes an open-source monitoring app that graphs your pulse in real time [9].

RED wire $=+3 \mathrm{~V}$ to $+5 \mathrm{~V}$

BLACK wire $=$ GND 
PURPLE wire $=$ Signal...... [9]

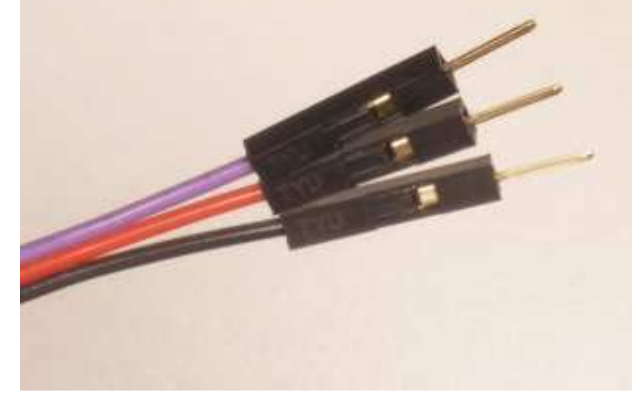

Fig. 2 Pulse sensor [9]

\section{Bread board}

This component is used because it will be the base of our device all the connections are being connected by this base [4].

\section{E. The ECG Sensor}

The ECG waveform is amplified, filtered and conditioned to a $1 \mathrm{Vpp}$ signal before applied to an Arduino or NodeMcu internal analog-to-digital converter (ADC) sample circuitry. The microcontroller gathers every five samples, adds an index frame number, a sampling rate and its checksum before sending the ECG signal over Bluetooth to a local tablet or smart phone running its

application. Frame transmission errors are supposed to be reduced, although errors may always occur despite the possible frame retransmissions [4].

\section{F. DS18B20}

$>$ The DS18B20-PAR uses a strict 1-Wire communication protocol to insure data integrity. Several signal types are defined by this protocol: reset pulse, presence pulse, write 0 , write 1 , read 0 , and read 1. All of these signals, with the exception of the presence pulse, are initiated by the bus master.

Multi-drop capability simplifies distributed temperature sensing applications

Requires no external components

$\pm 0.5^{\circ} \mathrm{C}$ accuracy from $-10^{\circ} \mathrm{C}$ to $+85^{\circ} \mathrm{C}$

Measures temperatures from $-55^{\circ} \mathrm{C}$ to $+100^{\circ} \mathrm{C}(-$ $67^{\circ} \mathrm{F}$ to $+212^{\circ} \mathrm{F}$ )

User-definable non-volatile temperature alarm settings

Alarm search command identifies and addresses devices whose temperature is outside of programmed limits (temperature alarm condition)

Ideal for use in remote sensing applications (e.g., temperature probes) that do not have a local power source [11].

\section{COMPARISON TABLE}

TABLE 1: Literature table

\section{Author}

1. Aboobacker sidheeque, Balamurugan .R, Deepak K .C , K. Sathish, "Heartbeat Sensing and Heart Attack Detection using Internet of Things: IoT", IJRASET, August 2016 , Volume:04, Issue: VIII [1].

2. Chaitali Kulkarni, Himani Karhade, Sonali Gupta, Prashant Bhende, Shital Bhandare, "Health Companion Device using IoT and Wearable Computing", IEEE- 2016 [3].

3. Carlo Alberto Boano, Matteo Lasagni, Kay R"omer, and Tanja Lange, "Accurate Temperature Measurements for Medical Research using Body Sensor Networks", IEEE - 2011 [4].

Parameters Limitation

Implementation

Check Heart beats and Detect heart attack

Emergency aid Android application

Check heart beats and body temperature Data store in cloud Emergency aid Generate monthly report of user. Accurate temperature measurements over long periods of time with a small node.
Only gives live heart beats and high as well as low levels of heart beat limit, not display heartbeats history of user.

Only check/detect heartbeats, not check other basic parameters of human health.

No android application

Wearable device: may create Yes overheating issue.

No self-organization.

No self-testing

Yes

No adaptive communication protocols 
International Journal of Trend in Scientific Research and Development (IJTSRD) ISSN: 2456-6470

\begin{tabular}{|c|c|c|c|}
\hline $\begin{array}{l}\text { 4. F.M. Machado, E.G. Bertogna } \\
\text { and M.A. Sovierzoski, "An IoT } \\
\text { Approach to an ECG Online } \\
\text { Monitor System in an Android } \\
\text { Application"[5]. }\end{array}$ & $\begin{array}{l}\text { Using Bluetooth } \\
\text { for communication } \\
\text { ECG signal is sent } \\
\text { remotely to the } \\
\text { cloud server }\end{array}$ & $\begin{array}{l}\text { Only ECG sensors. } \\
\text { If Bluetooth connection lost can't } \\
\text { predict value of ECG sensor. }\end{array}$ & No \\
\hline $\begin{array}{l}\text { 5. Pushpa Gothwal, Mieghem V C, } \\
\text { Sabbe M, Knockaert D, } \\
\text { "Designing of PPG based Heart- } \\
\text { beat Monitor", IJESC- } 2017 \text {, } \\
\text { Volume:07, Issue: } 4 \text { [6]. }\end{array}$ & $\begin{array}{l}\text { Designed and } \\
\text { tested the PPG } \\
\text { based heart beat } \\
\text { monitor system } \\
\text { using micro- } \\
\text { controller. } \\
\text { Result will display } \\
\text { on LCD. }\end{array}$ & $\begin{array}{l}\text { The subject movement can change the } \\
\text { output as well as the specific condition. }\end{array}$ & No \\
\hline
\end{tabular}

\section{Result Analysis}

The Result of proposed work will give us; Body Temperature, Heart rate, and ECG of human body and it will also give location of particular user which will be displayed on android application. So, we can get better result of human health in our hand at anywhere.

\section{Conclusion}

In this paper, the need for regular health monitoring is discussed and a Personal Health Monitoring device based on IOT that will monitor real time health status of the user is proposed. The solution comes with important features that contribute to: better health monitoring, fitness monitoring, secure data transfer, elimination of user's efforts to care for oneself, prealert system to help prevent critical condition.

\section{References}

1. Aboobacker sidheeque, Balamurugan .R, Deepak K .C , K. Sathish, "Heartbeat Sensing and Heart Attack Detection using Internet of Things: IoT", IJRASET, August 2016, Volume:04, Issue: VIII.

2. A. Butean, C. Buduleci, A. David, A. Daian, "Auxilum Medicine: a cloud based platform for real-time monitoring medical devices", 20th International Conference on Control Systems and Science, 2015.

3. Chaitali Kulkarni, Himani Karhade, Sonali Gupta, Prashant Bhende, Shital Bhandare, "Health Companion Device using IoT and Wearable Computing”, IEEE- 2016.
4. Carlo Alberto Boano, Matteo Lasagni, Kay R*omer, and Tanja Lange, "Accurate Temperature Measurements for Medical Research using Body Sensor Networks", IEEE - 2011.

5. F. M. Machado, E. G. Bertogna and M. A. Sovierzoski, "An IoT Approach to an ECG Online Monitor System in an Android Application", October $26^{\text {th }}-28^{\text {th }} / 201^{\text {th }}$ (C) Springer Nature Singapore Pte Ltd. 2017.

6. Pushpa Gothwal, Mieghem V C, Sabbe M, Knockaert D, "Designing of PPG based Heartbeat Monitor " , IJESC- 2017, Volume:07 , Issue: 4.

7. https://cdn-shop.adafruit.com/productfiles/2471/0A-ESP8266_Datasheet_EN_v4.3/

8. http://www.ti.com/product/LM35/datasheet/abstra ct\#SNIS1595780/

9. https://media.digikey.com/pdf/Data\%20Sheets/Pul se\%20PDFs/PulseSensorAmpedGettingStartedGui de.pdf/

10. Bandana Mallick, Ajit Kumar Patro, "Heart rate monitoring system using finger tip through arduino and processing software", IJSETR, January 2016, Volume:05, Issue: 1.

11. http://www.datasheetcafe.com/ds18b20-datasheettemperature-sensor/ 